\title{
Performance of ZigBee-Based wireless sensor nodes for real-time monitoring of fruit logistics
}

\author{
L. Ruiz-Garcia ，P. Barreiro , J.I. Robla \\ Laboratorio de Propiedades Fisicas y Tecnologias Avanzadas en Agroalimentacion, ETSI Agronomos, Universidad Politecnica de Madrid, \\ Avda. Complutense sin 28040 Madrid, Spain \\ Centro Nacional de Investigaciones Metalurgicas (CENIM-CSIC), Avda. Gregorio del Amo, 8, 28040, Madrid, Spain
}

\begin{abstract}
Progress in fruit logistics requires an increasing number of measurements to be performed in refrigerated chambers and during transport. Wireless sensor networks (WSN) are a promising solution in this field. This paper explores the potential of wireless sensor technology for monitoring fruit storage and transport conditions. It focuses in particular on ZigBee technology with special regard to two different commercial modules (Xbow and Xbee). The main contributions of the paper relate to the analysis of battery life under cooling conditions and the evaluation of the reliability of communications and measurements. Psychrometric equations were used for quick assessment of changes in the absolute water content of air, allowing estimation of future water loss, and detection of condensation on the product.
\end{abstract}

Keywords: Perishable products; Postharvest; Information technologies; Motes; Cold chain

\section{Introduction}

Fruits and vegetables are submitted to a variety of risks during transport and storage that are responsible for material quality losses. Among them are intrinsic biological and chemical processes that fresh produce undergoes after harvest, related to a lack of appropriate control on duration, temperature and humidity, which causes senescence and rot. As a consequence, effective cold-logistics monitoring is fundamental for ensuring product quality along the supply chain (Rodrfguez-Bermejo et al., 2007).

Wireless sensors networks (WSN) is a very promising technology in this field. A wireless sensor network is a system comprised of radio frequency (RF) transceivers, sensors, microcontrollers and power sources (Wang et al., 2006). Instrumented with a variety of sensors, such as temperature, humidity and volatile compound detection, WSN allow transport monitoring of perishable food products to be accomplished in a distributed way (Callaway, 2004).

The use of wireless intelligent sensors inside refrigerated vehicles was proposed in 2004 by Qingshan et al. (2004). Subsequently, Fuhr and Lau (2005) tested a RF device in a metal cargo container and demonstrated that it is possible to communicate with the outside world. Craddock and Stansfield (2005) proposed sensor fusion for the development of smart containers in order to improve security, gathering data from several sources in order to trigger the alarms. Containers may incorporate a variety of sensors to detect, identify, log and communicate what happens during their journeys around the world. Jedermann et al. (2006) presented a system for intelligent containers combining wireless sensor networks and RFID (Radio Frequency Identification). Ruiz-Garcia et al. (2007) analyzed monitoring intermodal refrigerated fruit transport, facing the integration of wireless sensor networks with multiplexed communications and fleet management systems. Such devices can be placed in transport vehicles in order 
to monitor the on-the-go environment and can be the basis for distributed systems, enabling environment sensing together with data processing. Behrens et al. (2007) studied the relation of battery lifetime and temperature in WSN, controlling the topology of the network in order to optimize energy-efficiency.

To date there has been no experimentation regarding fundamental factors in this field, such as node location inside the cargo, battery life and reliability of instrumentation under cooling conditions. Thus, experimentation in conventional refrigerated chambers could provide valuable information for near-future implementation in transports.

WSN can operate in a wide range of environments and provide advantages in cost, size, power, flexibility and distributed intelligence compared to wired ones. Wireless sensor networks offer permanent online access to the condition of freight. In a network, if a node cannot directly contact the base station, the message may be forwarded over multiple hops. By auto configuration set up, the network could continue to operate as nodes are moved, introduced or removed. Monitoring applications have been developed in medicine, agriculture, environment, military, machine/ building, toys, motion tracking and many other fields (Akyildiz et al., 2002; Baronti et al., 2007; Jedermann et al., 2006). Architectures for sensor networks have been changing greatly over the last 50 years, from the analogue 4-20 mA designs to the bus and network topology of today. Bus architectures reduce wiring and required communication bandwidth. Wireless sensors further decrease wiring needs, providing new opportunities for distributedintelligence architectures (Maxwell and Williamson, 2002; Wang et al., 2006).

For fieldbus architecture, the risk that cutting the bus that connects all the sensors persists. WSN eliminates all the problems arising from wires in the system. This is the most important advantage of using such technology for monitoring.

New miniaturized sensors and actuators based on microelectromechanical systems (MEMS) are in the development stage. Available MEMS include inertial, pressure, temperature, humidity, strain-gage, and various piezo and capacitive transducers for proximity, position, velocity, acceleration and vibration measurements (Wang et al., 2006). According to several research works, connecting wires to these devices can be more problematic than doing it by means of wireless designs (Jackson et al., 2008; Wise, 2007).

Another advantage for wireless sensor devices is the feasibility of installation in places where cabling is impossible, such as large concrete structures (Norris et al., 2008) or embedded within the cargo, which brings their readings closer to the true in situ properties of perishable products.

Wired networks are very reliable and stable communication systems for instruments and controls. However, wireless technology promises lower installation costs than wired devices, because required cabling engineering is very costly (Maxwell and Williamson, 2002; Wang et al., 2006).
At the current stage there are two available standard technologies for WSN: ZigBee and Bluetooth. Both are within the Industrial Scientific and Medical (ISM) band of $2.4 \mathrm{GHz}$, which provides license-free operations, huge spectrum allocation and worldwide compatibility. ZigBee is more suitable for WSN, mainly because of its low power consumption derived from its multi-hop communication. The power consumption in a sensor network is of primary importance and it should be extremely low. The ZigBee protocol places primary importance on power management. It has been developed to allow low power consumption and years of battery life. The suitability of this standard for monitoring has been proposed by various authors (Qingshan et al., 2004; Baker, 2005; Wang et al., 2006; Jedermann et al., 2006; Ruiz-Garcia et al., 2007).

Bluetooth works better in applications where large data rates are important, though it requires more energy (Shih et al., 2001). Bluetooth devices have lower battery life compared to ZigBee as a result of the processing and protocol management overhead which is required for ad hoc networking.

ZigBee provides higher network flexibility than Bluetooth, allowing different topologies such as star, cluster tree or mesh networks. ZigBee allows a larger number of nodes - more than 65,000 - according to specification. Transmission range is also longer $(1-100 \mathrm{~m})$ for ZigBee than for Bluetooth (1-10 m) (Baronti et al., 2007).

The main objective of this paper is to study the performance of ZigBee motes for monitoring the refrigerated conditions in fruit chambers with low temperatures, high humidity and different cargo densities. Reliability of communications and measurements, together with battery life, are major issues in this work.

\section{Materials and methods}

\subsection{The standard ZigBee and 802.15.4 characteristics}

ZigBee is an open specification that enables low power consumption, low cost and low data rate $(250 \mathrm{~kb} / \mathrm{s})$ for short-range wireless connections between various electronic devices. The ZigBee Alliance is an association of companies which develops standards and products for reliable, cost-effective, low power wireless networking. Major players in the electronics industry are members of the ZigBee Alliance (ZigBee Alliance, 2005).

The ZigBee standard is built on top of the IEEE 802.15.4 standard. The IEEE 802.15.4 standard defines the physical and MAC (Medium Access Control) layers for low-rate wireless personal area networks (IEEE, 2003). The physical layer supports three frequency bands with different gross data rates: $2450 \mathrm{MHz}(250 \mathrm{kbps})$, a $915 \mathrm{MHz}(40 \mathrm{kbps})$ and $868 \mathrm{MHz}(20 \mathrm{kbps})$. It also supports functionalities for channel selection, link quality estimation, energy measurement and clear channel assessment. ZigBee standardizes both the network and the application layer. The network layer is in charge of organizing and 
providing routing over a multi-hop network, specifying different network topologies: star, tree and peer to peer. The Application Layer provides a framework for distributed application development and communication.

\subsection{Commercial ZigBee motes}

Two different types of ZigBee motes have been used: Crossbow (Xbow) and Xbee-PRO (Xbee). In both systems, one sensor node (transmitter), and one base station (receiver), has been tested.

The Xbow motes are integrated by a microcontroller board (Micaz) together with an independent transducer board (MTS420) attached by means of a 52 pin connector (Fig. 1). The Micaz mote hosts an Atmel ATMEGA103/ 128L CPU running the Tiny Operating System (TinyOS) that enables it to execute programs developed using the nesC language. TinyOS was specifically developed for programming small devices with embedded microcontrollers. Micaz has a radio device Chipcon CC2420 $2.4 \mathrm{GHz}$ 250 kbps IEEE 802.15.4. The RF power in the Micaz can be set from $-24 \mathrm{dBm}$ to $0 \mathrm{dBm}$. Power is supplied by two AA alkaline batteries. For some of the experiments, two D type batteries were substituted.

The MTS420 board hosts a variety of sensors that can be easy removed: temperature and relative humidity (Sensirion SHT), light intensity (TAOS TSL2550D), barometric pressure (Intersema MS5534B), two-axis accelerometer (ADXL202JE) and GPS (Leadtek GPS-9546) that can be easy removed. A laptop computer is used as the receiver, and communicates with the nodes through a Micaz mounted on the MIB520 ZigBee/USB gateway board; this device also provides a USB programming interface. For this paper, only Sensirion SHT sensors were used.

The Xbee-PRO RF module is a ZigBee/IEEE 802.15.4 compliant solution for WSNs. Advanced configurations can be implemented using simple AT commands (Hayes command set). According to the manufacturer, it uses $60 \mathrm{~mW}(18 \mathrm{dBm}), 100 \mathrm{~mW}$ EIRP (Equivalent isotropically radiated power) power output (up to $1.6 \mathrm{~km}$ range).

Based on the Xbee-PRO development kit, we developed a prototype for monitoring. It includes an Xbee-PRO

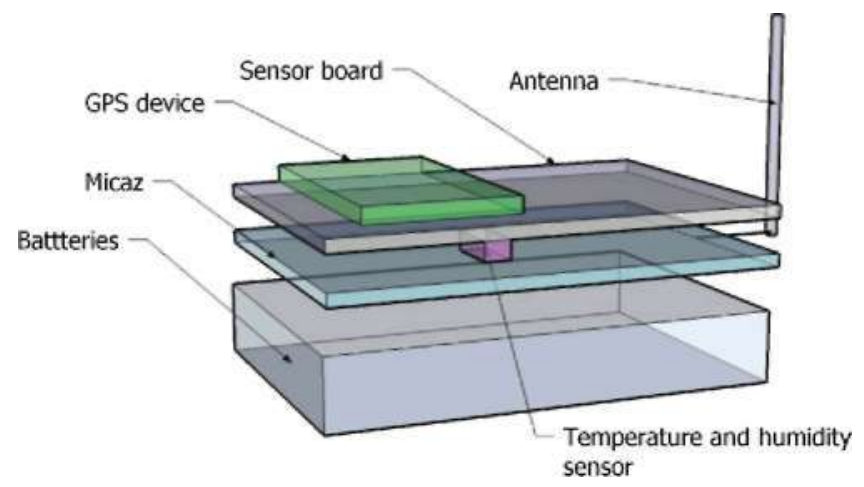

Fig. 1. Xbow mote. board, together with a development kit from a Sensirion SHT sensor; power for both came from a $12 \mathrm{~V} 7 \mathrm{Ah}$ battery. This sensor measures temperature and humidity, using CMOS (Complementary Metal Oxide Semiconductor) technology, and is the same sensor installed on the Xbow motes.

The SHT is a single-chip relative humidity and temperature multi-sensor module that delivers a calibrated digital output. The device includes a capacitive polymer sensing element for relative humidity and a bandgap temperature sensor. Both are seamlessly coupled to a 14-bit analog-todigital converter and a serial interface circuit on the same chip. Each SHT is individually calibrated in a precision humidity chamber. The calibration coefficients are programmed into the OTP (One Time Programmable) memory. These coefficients are used internally during measurements to calibrate the signals from the sensors.

For temperatures significantly different from $25{ }^{\circ} \mathrm{C}$, it is necessary to perform humidity compensation; the temperature coefficient of the RH sensor should be considered (Eqs. (1) and (2)).

$\mathrm{RH}_{\mathrm{me}} \mathrm{ar}=(-4)+0.0405 * \mathrm{SO}_{\mathrm{RH}}+\left(-2.8 * 10-{ }^{6}\right) * \mathrm{SOj}^{\wedge} \mathrm{H}$

SORH $=$ Sensor Output Relative Humidity

$\mathrm{RH}_{\text {true }}=\left(\mathrm{ro}_{\mathrm{C}}-25\right) *\left(0.01+0.00008 * \mathrm{SO}_{\mathrm{RH}}\right)+\mathrm{RH}_{\text {lmear }}$

Our Xbee-PRO based prototype represents an autonomous solution for wireless monitoring of temperature and humidity (Fig. 2).

\subsection{Experiments}

Two different types of experiments were conducted in order to verify the performance and reliability of ZigBee wireless nodes (see Table 1). Some were carried out in an experimental refrigerated chamber (ERC); the remainders were conducted in a commercial store (CWC) in the wholesale fruit and vegetable market in Madrid.

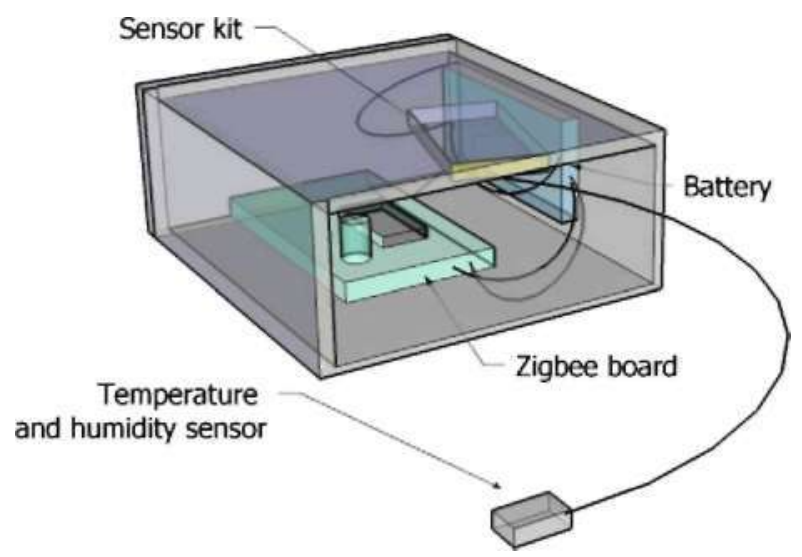

Fig. 2. Xbee-PRO based mote prototype. 
Table 1

Summary of experiments

\begin{tabular}{|c|c|c|c|c|c|}
\hline Experiment & Description & Mote type & Battery type & Sample rate/sensors & Set point \\
\hline \multirow[t]{3}{*}{ ERC } & Empty-chamber, three positions sampled & Xbow & Alcaline $2 *$ AA & $11 \mathrm{~s} T, \mathrm{RH}, \mathrm{GPS}$ & $0{ }^{\circ} \mathrm{C}, 8{ }^{\circ} \mathrm{C}, 20{ }^{\circ} \mathrm{C}$ \\
\hline & & & Alcaline $2 * \mathrm{D}$ & 11 s $T$, RH, GPS & $0^{\circ} \mathrm{C}, 8^{\circ} \mathrm{C}, 20^{\circ} \mathrm{C}$ \\
\hline & $\begin{array}{l}\text { Chamber loaded with } 7201 \text { of water, } \\
\text { sampled inside and outside pallets }\end{array}$ & Xbow & Alcaline $2 *$ AA & $\begin{array}{l}11 \mathrm{~s} T, \mathrm{RH} \\
\text { with and without GPS }\end{array}$ & $0^{\circ} \mathrm{C}$ \\
\hline \multirow[t]{2}{*}{$\mathrm{CWC}$} & $\begin{array}{l}\text { Chamber loaded with } 13 \text { pallets of chard, } \\
\text { three positions sampled }\end{array}$ & Xbow & Alcaline $2 * \mathrm{D}$ & $11 \mathrm{~s} T, \mathrm{RH}, \mathrm{GPS}$ & $3{ }^{\circ} \mathrm{C}$ \\
\hline & & Xbee & Lead $12 \mathrm{~V} 6 \mathrm{Ah}$ & $10 \mathrm{~s} T, \mathrm{RH}$ & \\
\hline
\end{tabular}

For both experiments, the main parameters considered were the ratio of measurement losses $(\%)$, battery life (minimum), and the influence of node location and on/off operation of the cooling system. The reliability of measurements in relation to battery status was also considered.

The ERC has a capacity of $5.98 \mathrm{~m}^{3}$, and is made of metallic panels, with two engines. The WSN motes have been tested at the ERC with two battery types, two cargo levels, three different set points and at several locations inside the chamber (see Table 1).

Since temperature $(T)$ and relative humidity $(\mathrm{RH})$ conditions for fresh fruit during transport ranges from $-0.5{ }^{\circ} \mathrm{C}$ to $12.2{ }^{\circ} \mathrm{C}$ and from $75 \%$ to $90 \% \mathrm{RH}$ (see Table 2), in this study three different conditions within this range were selected: ambient conditions $\left(20{ }^{\circ} \mathrm{C}\right.$ approx. $), 8{ }^{\circ} \mathrm{C}$ with $65 \% \mathrm{RH}$ and $0{ }^{\circ} \mathrm{C}$ with $90 \% \mathrm{RH}$ (see Table 1). This third situation corresponds to the optimal conditions for transporting many species such peaches or strawberries (see Table 2) (GDV, 2005).

The influence of cargo density on communications reliability was evaluated at the ERC, comparing transmission through empty-chamber with regard to nodes located inside/outside a pallet full of water bottles (720 1).

Experiments performed at CWC $\left(1848 \mathrm{~m}^{3}\right)$ make use of a chamber that provides on/off glycol cooling and which is insulated with polyurethane foam sandwiched between two layers of corrugated plate (total wall thickness is $0.16 \mathrm{~m}$ ). The set point for this chamber was fixed at $3{ }^{\circ} \mathrm{C}$ by user restrictions. Tests were also conducted to measure the effect of different cargo densities, and to compare several ZigBee systems (Xbow and Xbee) under three different conditions: free space, 13 pallets full of boxes between the emitter and receiver, and emitter inside the sixth pallet in a line of 13 . The second and the third situations simulate the implemen-

Table 2

Optimal temperature and relative humidity conditions for some fresh fruits during transport (GDV, 2005)

Type of cargo

$\begin{array}{ll}\begin{array}{l}\text { Transport } \\ \text { temperature }\left({ }^{\circ} \mathrm{C}\right)\end{array} & \begin{array}{l}\text { Relative } \\ \text { humidity }(\%) \\ 10.0-12.0\end{array} \\ \begin{array}{l}85-90 \\ 4.5\end{array} & 85-90 \\ -0.5-1.5 & 85-90\end{array}$

Pineapple, bananas, mangoes, $\quad 10.0-12.0 \quad 85-90$ melons, lemons

Oranges $\quad 4.5 \quad 85-90$

Apples, apricots, pears, strawberries, $-0.5-1.5 \quad 85-90$ cherries, peaches, grapes tation of wireless nodes inside refrigerated trailers, where the normal cargo situation is two lines of 13 Pallet EUR $2(1 \times 1.2 \mathrm{~m})$ or three lines of 11 Pallet EUR $(0.8 \times 1.20 \mathrm{~m})(\mathrm{ISO}, 2003)$. During the experiments at the CWC, there was approximately one meter between the pallets and the walls.

The program installed in the motes collects data from all the sensors at a fixed sample rate $(11 \mathrm{~s}$ for Xbow; $10 \mathrm{~s}$ for Xbee), with each transmission referred to as a "packet". Sample rate (SR) was fixed to provide very limiting conditions for battery life, a major issue in this study. In all the experiments, the RF power in the Xbow motes was set to OdBm.

\subsection{Data analysis}

A specialized MATLAB program has been developed for assessing the percentage of lost packets (\%) in transmission, by means of computing the number of multiple sending failures. A multiple failure of $m$ messages occurs whenever the elapsed time between two messages lies between $1.5 \times m \times$ SR and $2.5 \times m \times$ SR. For example, with a sample rate of $11 \mathrm{~s}$, a single failure $(m-1)$ occurs whenever the time period between consecutives packets is longer than $16.5 \mathrm{~s}(1.5 \times 1 \times 11)$ and shorter than $27.5 \mathrm{~s}(2.5 \times 1 \times$ 11). The total number of lost packets is computed based on the frequency of each failure type. Accordingly, the total percentage of lost packets is calculated as the ratio between the total number of lost packets and the number of sent packets.

The standard error (SE) associated to the ratio of lost packets is computed based on a binomial distribution as expressed in Eq. (3), where $n$ is the total number of packets sent, and $p$ is the ratio of lost packets in the experiment.

$\mathrm{SE}=\underset{\mathrm{V}}{J P} \boldsymbol{n}-P L$

\subsection{Analysis of variance}

Analysis of variance (ANOVA) has been performed in order to evaluate the effect of temperature on battery life. ANOVA allows partitioning of the observed variance into components due to different explanatory variables. The software STATISTICA (StatSoft, Inc.) was used for this purpose (Devore and Farnum, 2004). 
Table 3

Coefficients used to compute the psychometric data (ASABE, 2006). According to Eqs. (3)-(5)

$$
\begin{aligned}
& R=22,105,649.25 \\
& A=-27,405.526 \\
& 5=97.5413 \\
& \mathrm{C}=-0.146244
\end{aligned}
$$

$$
\begin{aligned}
D & =0.12558 \times 10 \sim^{3} \\
E & =-0.48502 \times 10 \sim^{7} \\
F & =4.34903 \\
G & =0.39381 \times 10 \sim^{2}
\end{aligned}
$$

\subsection{Psychrometric data}

The ASAE D271.2, denned in April 1979 and reviewed in 2005, is used for computing the psychrometric properties of air at CWC experiments (ASABE, 2006). Eqs. (4)-(6) and Table 3 enable the calculation of all psychrometric data of air whenever two independent psychrometric properties of an air-water vapor mixture are known in addition to the atmospheric pressure $\left(10^{5} \mathrm{~Pa}\right)$.

Ps $=R * \mathrm{e} \quad F, T-G, T I$

where Tis the temperature $(\mathrm{K})$, Ps is the saturation vapour pressure (Pa) (ASABE, 2006).

${ }^{P v}={ }^{P s}$ ToO

where $\mathrm{Pv}$ is the vapor pressure (Pa) (ASABE, 2006).

$H=\begin{aligned} & 0.6219 * \mathrm{PV} \\ & \mathrm{P} \text { at m- }-\mathrm{PV}_{\mathrm{V}}\end{aligned}$

where //is the absolute humidity ( $\mathrm{g} / \mathrm{kg}$ dry air), Patm is the atmospheric pressure (Pa) (ASABE, 2006).

\section{Results}

Tables 4 and 5 summarize the main results for the ERC and CWC experiments respectively. Results have been categorized into battery life assessment and communication and data reliability and will be presented accordingly in the text.

\subsection{Battery life}

Fig. 3 shows that battery life is clearly affected by temperature. Fisher's $F(F-19.9)$ shows that temperature
Table 5

Average percent of packet looses and corresponding standard error for Xbow and Xbee motes at CWC

$\begin{array}{lll}\text { Transmitter location } & \text { Xbow } & \text { Xbee } \\ \text { Empty room } & 1.38 \% \pm 0.06(34160) & 0.00 \pm 0.00(16906) \\ \text { Trough 13 pallets } & 1.92 \% \pm 0.07(39225) & 0.02 \pm 0.01(18325) \\ \text { Inside the boxes (pallet 6) } & 4.74 \% \pm 0.15(36614) & 0.26 \pm 0.06(15103)\end{array}$

Number of measurements is included in brackets.

has a significance in battery life. Thus for AA batteries the duration decreases from $610 \pm 83 \mathrm{~min}$ at $20{ }^{\circ} \mathrm{C}$ to $407 \pm 58$ at $8{ }^{\circ} \mathrm{C}$ and to $297 \pm 44 \mathrm{~min}$ at $0{ }^{\circ} \mathrm{C}$. Life for $\mathrm{D}$ type is on average $70 \%$ greater than for AA batteries, also with duration decreasing according to temperature from $655 \pm 145 \mathrm{~min}$ at $8{ }^{\circ} \mathrm{C}$ to $379 \pm 136 \mathrm{~min}$ at $0{ }^{\circ} \mathrm{C}$ (Fig. 4 ).

It is important to note that battery life for $\mathrm{Xbow}$ motes without GPS is extended up to $4500 \mathrm{~min}$ at $0{ }^{\circ} \mathrm{C}$ with $2 \mathrm{AA}$ batteries, while it falls below 300 min when a GPS device is mounted. Such heavy power consumption will be further discussed in relation to heat dissipation.

\subsection{Communication reliability}

In the experiments carried out at ERC, the percentage of lost packets was always higher at $8{ }^{\circ} \mathrm{C}(2.15-15.73 \%)$ than at $0{ }^{\circ} \mathrm{C}$ (below $1 \%$ ) or $20{ }^{\circ} \mathrm{C}$ (below $0.27 \%$ Table 4 ). This fact seems to be related with the number of on/off operations of the cooling system: none for $20^{\circ} \mathrm{C}, 1$ for $0{ }^{\circ} \mathrm{C}$ and between 15 and 19 for $8{ }^{\circ} \mathrm{C}$.

Table 5 shows the results obtained for the experiments conducted at CWC. Xbee motes performed better than Xbow in terms of reliability of communications; a lower ratio of lost packets was found at all locations for Xbee compared to Xbow. In both cases (Xbow and Xbee), no restrictions were found for signal propagation along 13 pallets, even though the rate of lost packets was higher in this situation compared to an empty room. The highest ratio of lost packets was found when the mote was located inside the cargo (emitter inside pallet 6), reaching as high as $4.74 \%$ for Xbow motes. Note that this value is lower than the $15 \%$ found for ERC; the greater amount of free space

Table 4

Average percentage of packet looses for Xbow motes in ERC and corresponding standard error

Transmitter location

$\begin{array}{ll} & \text { Off } \\ & 20{ }^{\circ} \mathrm{C} \\ \text { Lower corner } & 0.27 \% \pm 0.06 \% \\ & (7256)^{\mathrm{a}} \\ & 0^{\mathrm{b}} \\ 60 \mathrm{~cm} \text { over the opposite corner } & 0.00 \% \pm 0.00 \% \\ & (5482)^{\mathrm{a}} \\ 60 \mathrm{~cm} \text { over the corner bottom } & 0^{\mathrm{b}} \\ & 0.00 \% \pm 0.00 \% \\ & (7180)^{\mathrm{a}} \\ & 0^{\mathrm{b}}\end{array}$

a Number of measurements.

b Number of on/off control cooling.
On

$$
\begin{aligned}
& !^{\circ} \mathrm{C} 65 \% \mathrm{RH} \\
& 15.73 \% \pm 0.52 \% \\
& (4849)^{\mathrm{a}} \\
& 18^{\mathrm{b}} \\
& 4.63 \% \pm 0.31 \% \\
& (4515)^{\mathrm{a}} \\
& 19^{\mathrm{b}} \\
& 2.15 \% \pm 0.23 \% \\
& (3848)^{\mathrm{a}} \\
& 15^{\mathrm{b}}
\end{aligned}
$$

$0{ }^{\circ} \mathrm{C} 90 \% \mathrm{RH}$

$0.50 \% \pm 0.12 \%$ $(3735)^{\mathrm{a}}$

$1^{\mathrm{b}}$

$1.00 \% \pm 0.18 \%$ $(2876)^{\mathrm{a}}$ $1^{\mathrm{b}}$

$0.00 \% \pm 0.0 \%$ $(3149)^{\mathrm{a}}$ $1^{\mathrm{b}}$ 


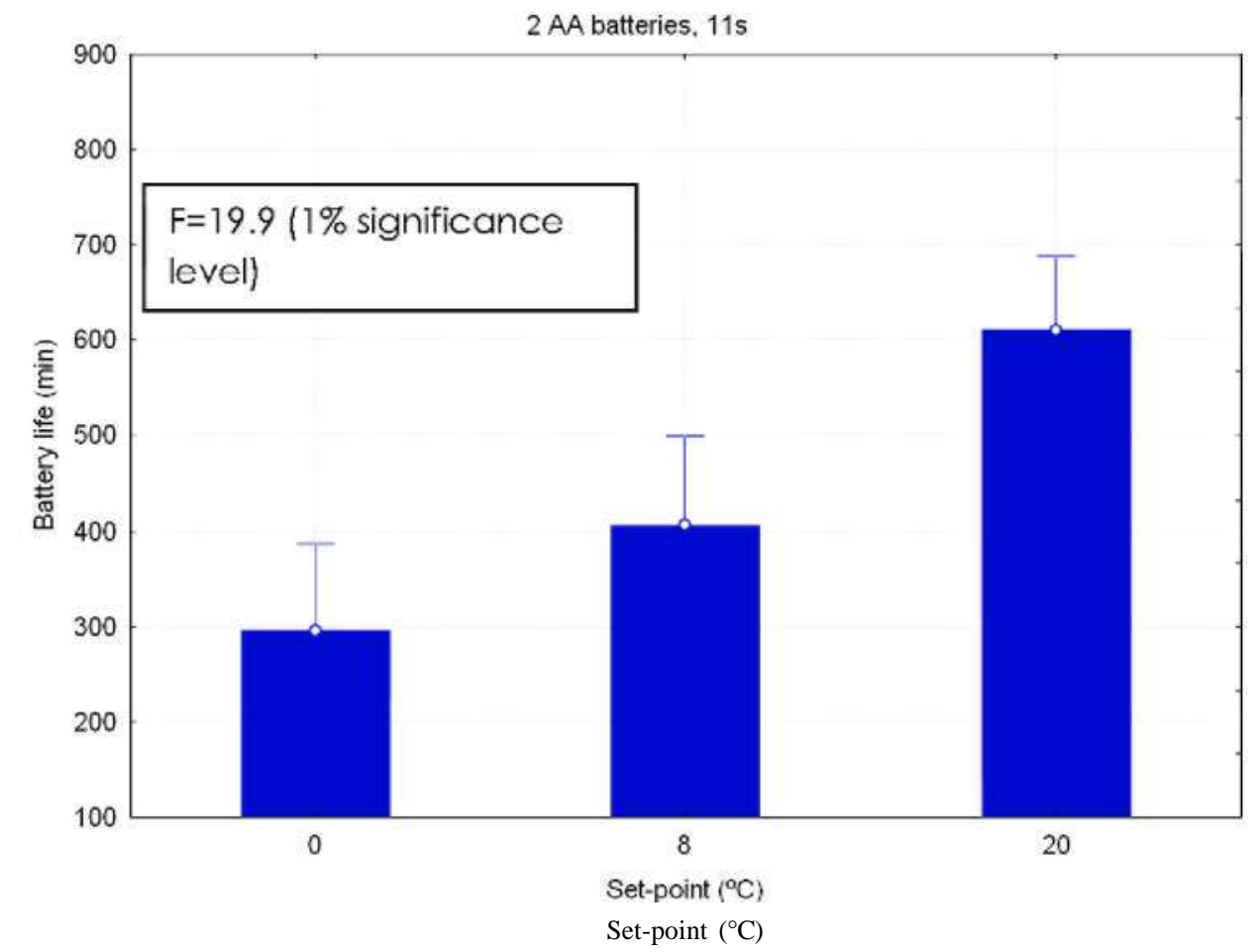

Fig. 3. Average battery life for different set-points during the experiments in ERC for 2AA batteries at $11 \mathrm{~s}$ sample rate.

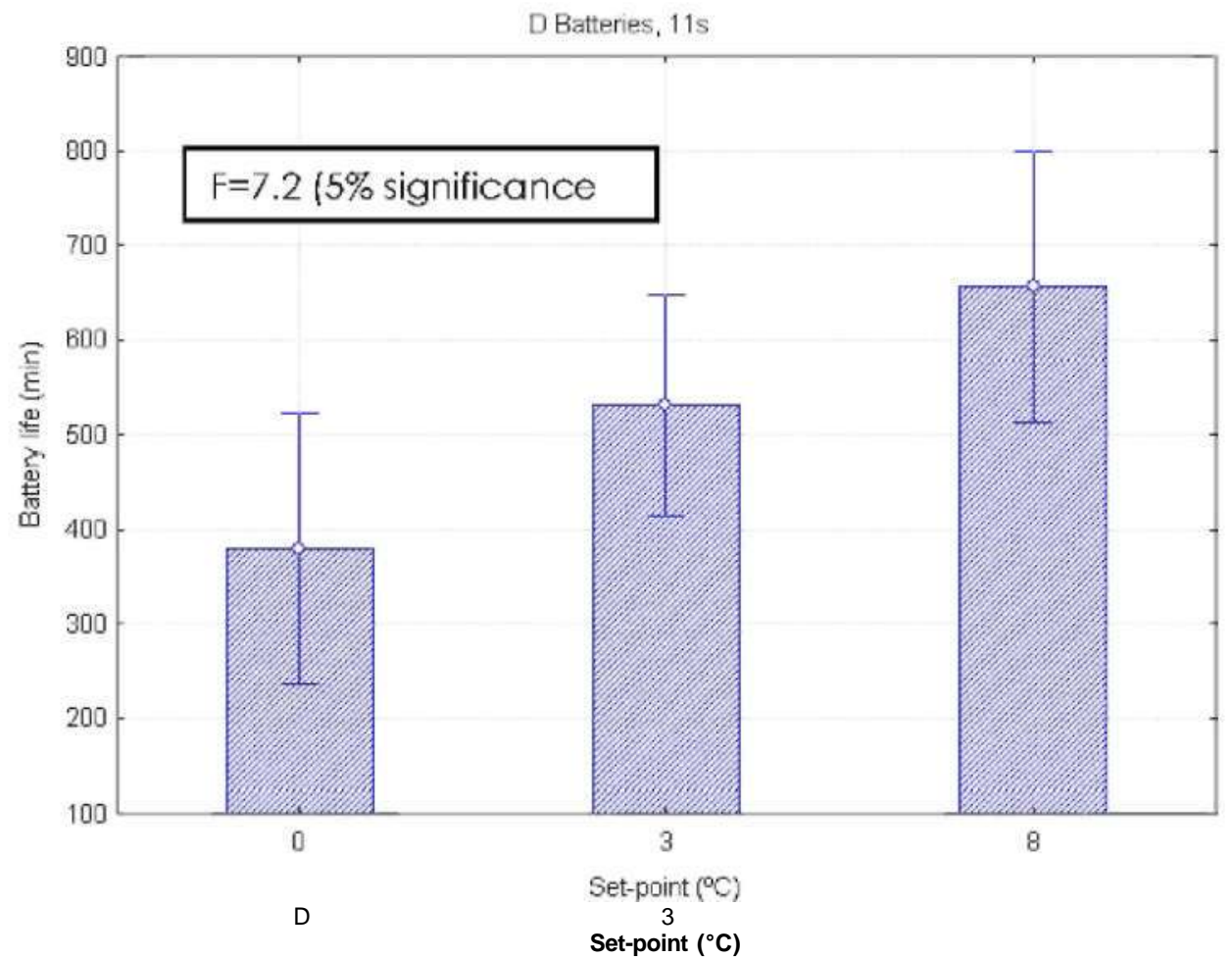

Fig. 4. Average battery life for different set-points during the experiments in CWC with $2 \mathrm{D}$ batteries at $11 \mathrm{~s}$ sample rate.

at CWC compared to ERC could be the basis of such a difference.

The ratio and manner in which data packets were lost was not the same for the two systems (Xbow and Xbee). In our experiments, Xbee motes always lost fewer packets than Xbow motes. In the Xbee motes, lost packets were distributed regularly whenever the emitter is inside the cargo (a feature which is also congruent with the Xbow motes). However, in Xbow motes, a high accumulation of packets were found at certain periods $(\mathrm{a}, \mathrm{b}, \mathrm{c}$ in Fig. 5) which may be related to the movement of objects or persons inside the chamber. This was not found for 

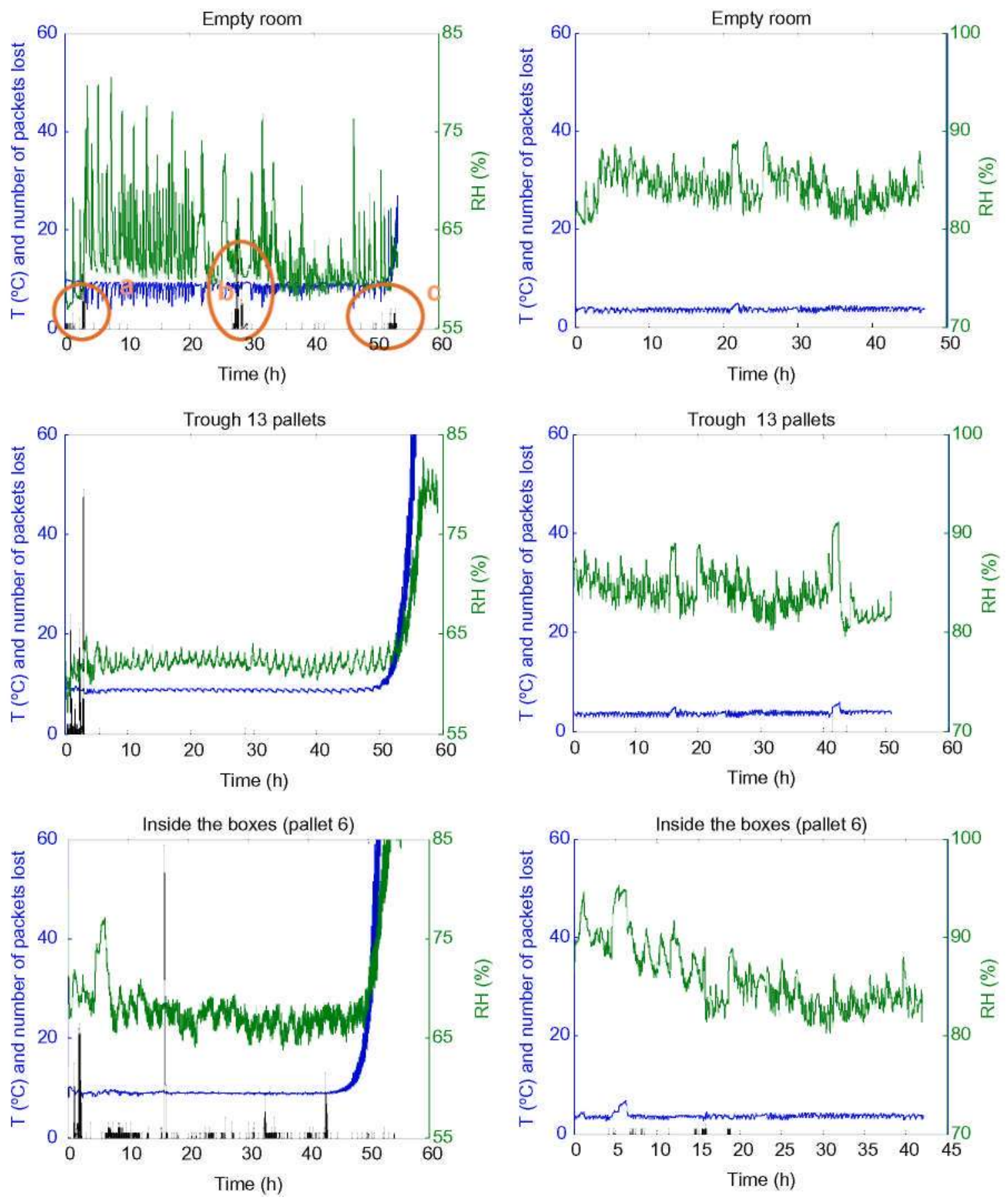

Fig. 5. RH (\%), $T\left({ }^{\circ} \mathrm{C}\right)$ number of data lost packets for Xbow (left column) and Xbee (right column) in CWC; a, b and c stands for periods of accumulation of lost packets.

Xbee motes, even though the receiver was connected in both cases to the same PC.

\subsection{Data reliability}

Data reliability is a critical issue for ensuring interest in future implementations of this technology within transportation.

Fig. 5 compares the time evolution of $T\left({ }^{\circ} \mathrm{C}\right)$ and $\mathrm{RH}$ (\%) for Xbow and Xbee motes at CWC experiments. Gen- erally $\mathrm{RH}$ varies inversely to $T$; only for door openings were there a simultaneous increase in $T$ and $\mathrm{RH}$ due to hot air entering from outside. A major difference between Xbow and Xbee concerns the order of magnitude in $\mathrm{T}$ and RH which stayed around $8{ }^{\circ} \mathrm{C}$ and $60 \% \mathrm{RH}$ for Xbow while being around $3.5{ }^{\circ} \mathrm{C}$ and $85 \%$ for Xbee (set-point of the chamber was $3{ }^{\circ} \mathrm{C}$ ). This is a puzzling question since both sensors are the same and came calibrated from the manufacturer. This issue seems to be related to the position of the $T$ and RH sensor. In the case of Xbow, the sensor is 

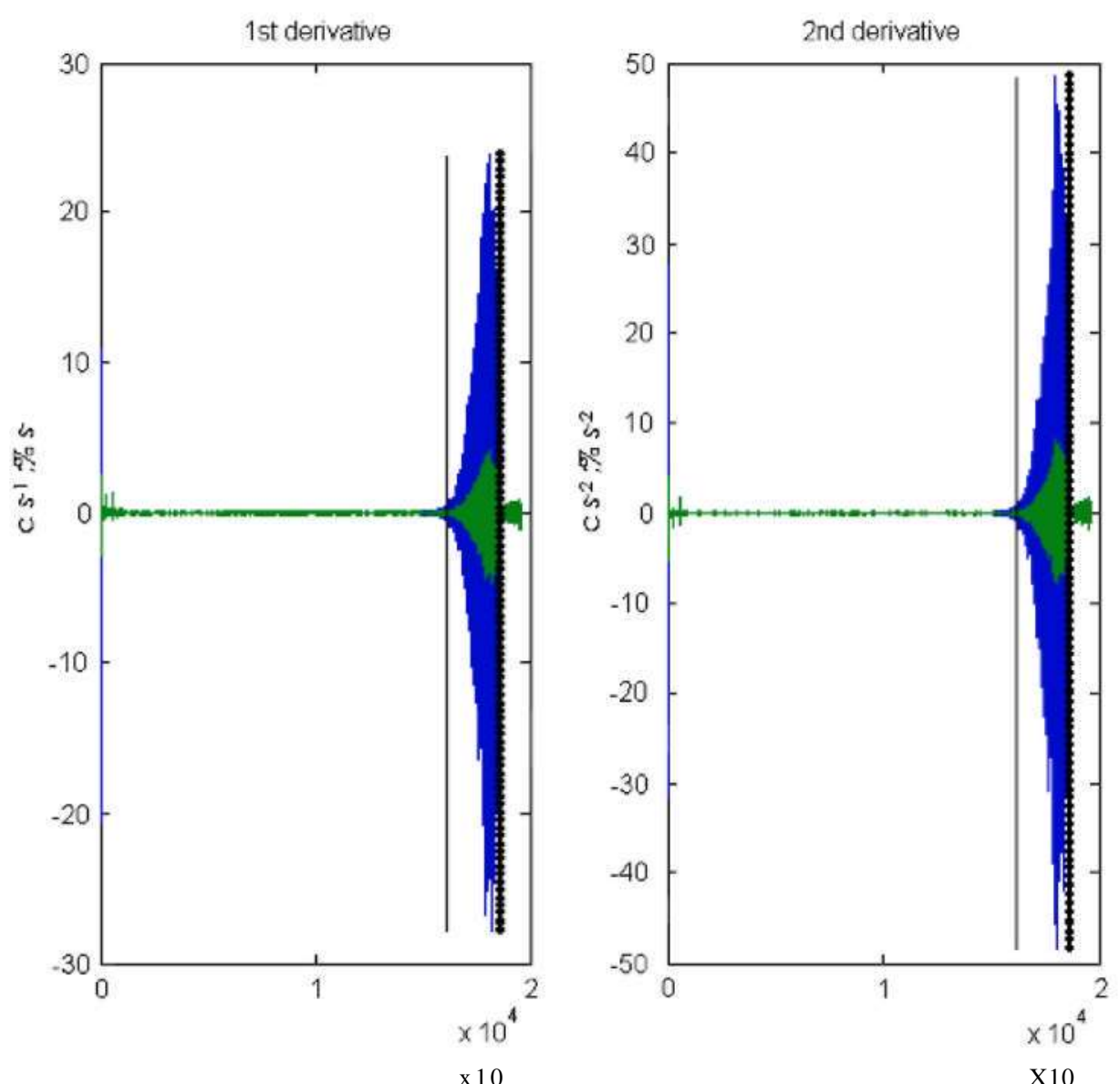

Fig. 6. First and second derivative for Xbow temperature data in CWC. The wide increase is oscillation that battery level is getting to low.

located just under the GPS electronics (Fig. 1) which has been shown to be highly battery-consuming and, accordingly, a significant heat source. In contrast, the Xbee the sensor is isolated at the end of a cable (see Fig. 2).

At high $T$, RH decreases and that would explain the lower order of magnitude for $\mathrm{RH}$ at Xbow compared to Xbee.

The high energy consumption of the GPS could be the cause of heat dissipation and the $T$ increase for the Xbow mote.

Fig. 5 shows that for the Xbow motes, $T\left({ }^{\circ} \mathrm{C}\right)$ and $\mathrm{RH}$ (\%) measurements become erroneous at low battery voltage. This occurred around $2160 \mathrm{mV}$ in all experiments. $T$ $\left({ }^{\circ} \mathrm{C}\right)$ rises enormously and both $\mathrm{RH}(\%)$ and $T\left({ }^{\circ} \mathrm{C}\right)$ increase in variability. First and second derivatives of $T\left({ }^{\circ} \mathrm{C}\right)$ and RH (\%) allow addressing abnormal fluctuations of measurements as an indication of initial failure (Fig. 6).

Table 6 indicates the battery voltage threshold at which $\mathrm{T}$ and $\mathrm{RH}$ measurements lost their reliability (between 2159 and $2167 \mathrm{mV}$ ). Table 7 compares the average and standard deviation of $T$ and RH for both Xbow and Xbee motes.

\subsection{Psychrometry}

For the data registered in the CWC with Xbow and Xbee motes, the absolute humidity of air was calculated
Table 6

Battery conditions for abnormal $T$, HR measurements with Xbow motes in CWC

\begin{tabular}{lllll} 
& \multicolumn{2}{l}{$\begin{array}{l}\text { Parameters at initial } \\
\text { failure }\end{array}$} & $\begin{array}{l}\text { Parameters at failure } \\
\text { stabilization }\end{array}$ \\
& $\begin{array}{l}\text { Battery } \\
\text { life }(\mathrm{h})\end{array}$ & $\begin{array}{l}\text { Voltage } \\
(\mathrm{mV})\end{array}$ & $\begin{array}{l}\text { Battery } \\
\text { life }(\mathrm{h})\end{array}$ & $\begin{array}{l}\text { Voltage } \\
(\mathrm{mV})\end{array}$ \\
Empty room & 51.2 & 2163.0 & - & - \\
Trough 13 pallets & 49.9 & 2166.7 & 57.5 & 2094.2 \\
Inside pallet 6 & 46.6 & 2159.2 & 54 & 2070
\end{tabular}

Table 7

Comparison of average $\mathrm{RH}(\%)$ and $T\left({ }^{\circ} \mathrm{C}\right)$ between Xbow and Xbee motes

\begin{tabular}{lllll} 
& \multicolumn{2}{l}{ Mean RH $(\%)$} & \multicolumn{2}{l}{ Mean $T\left({ }^{\circ} \mathrm{C}\right)$} \\
& Xbow & Xbee & Xbow & Xbee \\
Empty room & $62.6 \pm 3.8$ & $84.3 \pm 1.7$ & $8.40 \pm 1.1$ & $3.60 \pm 0.3$ \\
Trough 13 pallets & $62.1 \pm 0.7$ & $84.1 \pm 2.1$ & $8.86 \pm 0.5$ & $3.71 \pm 0.4$ \\
Inside pallet 6 & $67.6 \pm 2.1$ & $86.0 \pm 3.3$ & $9.06 \pm 0.3$ & $3.68 \pm 0.5$
\end{tabular}

based on the ASAE standard D271.2 (1979), (ASABE, 2006).

Psychrometric charts are included in Figs. 7 and 8, which illustrate the evolution of air absolute humidity ( $H, \mathrm{~kg}$ of water $/ \mathrm{kg}$ of dry air) related to the $T\left({ }^{\circ} \mathrm{C}\right)$ for 

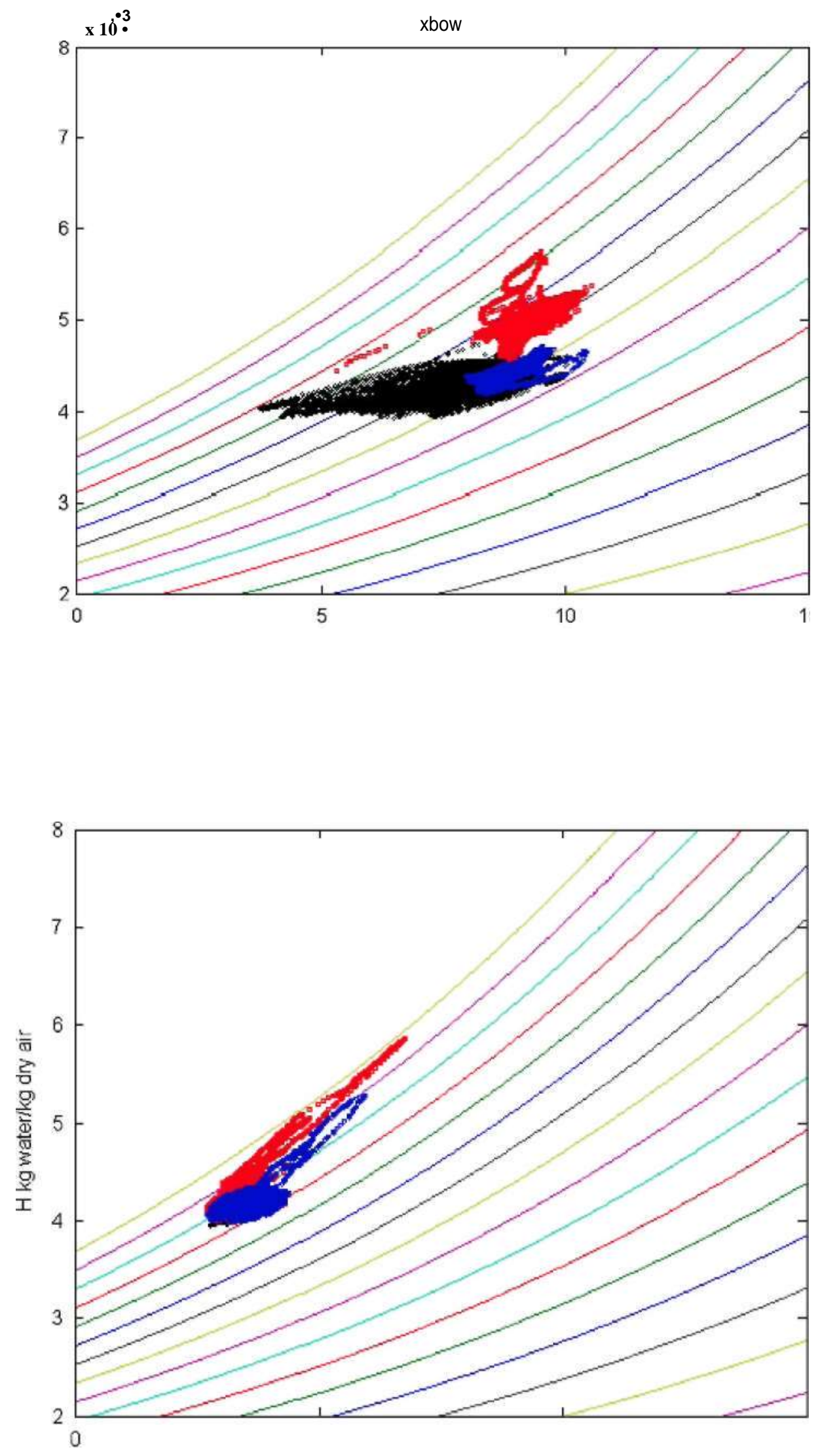
empty room, 13 pallet and emitter-inside-cargo experiments. Door opening created a great increment in $T\left({ }^{\circ} \mathrm{C}\right)$ and absolute humidity, which then returns to normal again once the door is shut. During the rest of the time, it is also possible to detect the interaction between air properties and the product; with the cycles of cooling, variations in the absolute humidity can be estimated: condensation over the products (as loss of absolute humidity), or water evaporation (as an increase in absolute air humidity). The lines refer to the absolute water content for several values of $\mathrm{RH}$ $(100-45 \%)$. It can be seen in Fig. 7 that temperature varies more with the empty-chamber because of higher thermal inertia.

The psychrometric data for Xbow motes (Fig. 7) shows a clear segregation between the three positions inside the chamber. Black dots represent air conditions with emptychamber, blue motes refer to data from a cool loaded-room and red data represent ambient data inside the pallet. Air absolute humidity inside the pallet is always higher than air outside cargo, which indicates water loss from the product.

With empty-chamber, air temperature inertia seems to be lower than for loaded-room and thus temperature variability is much higher; for this same situation, absolute humidity has lower variation.

The psychrometric chart for Xbee motes (Fig. 8) shows empty-chamber data to be indistinguishable from loadedroom data. This fact may be due to the location where the sensor was situated, at the end of the cable and touching the floor of the room. As before, air absolute humidity inside the cargo is higher than for other situations, due to water loss in the product. In these types of motes - where the sensor is located far from the electronics - a clear pattern in temperature and air humidity is found which corresponds to door opening of the chamber (simultaneous increase of temperature and absolute humidity). This pattern is different for Xbow motes due to the location of the sensor (beneath the GPS device) and thus very much affected by the heat dissipating from the electronics.

Distributed information of $T$, RH and absolute humidity may be used to address water loss or condensation along the cargo providing valuable information to improve the control algorithms of the cooling and ventilation system.

\section{Conclusions}

In this paper, the feasibility of using two types of wireless nodes (Xbee and Xbow) for monitoring storage and transport was experimentally assessed. Both ZigBee motes perform adequately under typical $T$ and RH conditions in the cold supply chain. The suitability of this technology for monitoring refrigerated chambers as well as the implementation under transport conditions has been demonstrated. This kind of system can be used in a warehouse, container or vehicle for remotely monitoring and tracking environmental characteristics, geographical locations of assets such as a shipping container and content and for communication with gateway of a network e.g. Internet, cellular network and satellite network. These sensors can be placed in locations usually not accessible for other systems.

Battery life decreases under cooling conditions. For $2 *$ AA batteries in a GPS Xbow mote, life at $0{ }^{\circ} \mathrm{C}$ $(297 \pm 44 \mathrm{~min})$ is half than that at $20^{\circ} \mathrm{C}(610 \pm 83 \mathrm{~min})$, increasing to $379 \pm 136 \mathrm{~min}$ for $2 \mathrm{D}$ batteries at $0{ }^{\circ} \mathrm{C}$. When the GPS device is removed from the mote, battery life is extended by a factor of 10 .

Measurements for Xbow sensors become erroneous when the battery voltage is less than $2160 \mathrm{mV}$ regarding to $3000 \mathrm{mV}$ corresponding to full charge. Further research is necessary, for programming algorithms in order to save energy and to extend battery life. The on-board identification of erroneous measurements is basic for commercial purposes and has been outlined in this paper.

Xbee motes could be a good solution for wireless monitoring in refrigerated industrial environments, because the rate of lost packets inside the cargo $(0.26 \%)$ is always lower than that of Xbow (4.74\%). For the latter, a large quantity of lost packets is found at singular moments, which never occur for Xbee motes. Wireless communications can be affected by the interference induced by motors of the cooling equipment. This interference is higher for Xbow compared to Xbee motes, but further tests are required to evaluate the effect of this factor. The better reliability of the Xbee motes corresponds with their higher RF power. However, a potential concern in the Xbee based prototype is the large battery size, which makes the system much bigger than the Xbow motes.

The performance of the system can be improved by the implementation of advanced network topologies, such as point-to-multipoint, peer-to-peer and mesh, improving the reliability and robustness of the system. It is important to optimize the performance of every component (sensors, microcontrollers and radiofrequency devices) to consume as little power as possible while still meeting the requirements of the application in terms of data throughput, latency and reliability.

There is a need for testing the long-term behavior of the systems in real-world fruit transports. Other issues such as signal processing, powering, communication, location, orientation data storage and computation capabilities also need to be addressed, and are currently being investigated by the authors.

Another important topic is fault detection and isolation. The detection of failures in a wireless network is fundamental. For Xbow motes, automated detection of erroneous measurements is addressed on the basis of abnormal oscillations of measurement. A large effect of the GPS device on dissipation and temperature measurements is found whenever the $T$ and $\mathrm{RH}$ sensor is not properly located in the mote.

The use of psychrometric equations has been implemented for quick assessment of changes in the absolute water content of air. A multi-distributed system that 
implements this type of sensors can estimate water loss from the products using the psychrometric model, and also to detect condensation on the commodities.

\section{Acknowledgements}

Thanks to the SENSOFRIGO research program supported by the Ministry of Education and Science of Spain (Ref: AGL2003-06073-C02-01) and TAGRALIA project funded by the Comunidad de Madrid. The authors would like to thank also Alejandro Navarro, Pablo Poncela and Jorge Sanchez for their help in the experiments.

\section{References}

Akyildiz, F., Su, W., Sankarasubramaniam, Y., Cayirci, E., 2002. Wireless sensor networks: a survey. Computer Networks 38, 393-422.

ASABE Standards, 2006. Psychrometric data ASAE D271.2 APR1979, R2005, St. Joseph, MI, Disponible en. <www.asabe.org> (January 2007).

Baker, N., 2005. ZigBee and Bluetooth. IEEE Computing and Control Engineering 200 (April/May), 20-25.

Baronti, P., Prashant, P., Chook, V.W.C., Chessa, S., Gotta, A., Fun Hu, Y., 2007. Wireless sensor networks: wireless sensor networks: a survey on the state of the art and the 802.15.4 and ZigBee standards. Computer Communications 30 (7) (May), 1655-1695.

Behrens, C, Bischoff, O., Lueders, M., Laur, R., 2007. Energy-efficient topology control for wireless sensor networks using online battery monitoring. In: Langenberg, Karl-Jorg (Ed.), Kleinheubacher Tagung 2006. U.R.S.I. Landesausschuss in der Bundesrepublik Deutschland e.V, Kassel.

Callaway, E.H., 2004. Wireless Sensor Networks: Architectures and Protocols. Auerbach Publications, USA, 342pp.

Craddock, R.J., Stansfield, E.V., 2005. Sensor fusion for smart containers. In: IEE Seminar on Signal Processing Solutions for Homeland Security (2005/11108), London, UK, 11 October 2005, p. 5. ISB N0 863415644.

Devore, J.L., Farnum, N.R., 2004. Applied Statistics for Engineers and Scientists. Duxbury Press. ISBN-10:0534467199; ISBN-13:9780534467197.

Fuhr, P., Lau, R., 2005. Mesh radio network performance in cargo containers. Sensors Magazine Online, <www.sensorsmag.com/articles/ 0305/13/main.shtml> (10.09.06).

GDV, 2005. Container Handbook - Cargo Loss Prevention from German Marine Insurers. German Insurance Association. Gesamtverband der
Deutschen Versicherungswirtschaft E.V. <www.containerhandbuch.de> (03.02.06).

IEEE, 2003. IEEE Standard 802.15.4. Wireless Medium Access Control (MAC) and Physical Layer (PHY) Specifications for Low-Rate Wireless Personal Area Networks, 2003. <http://standards.ieee.org/ getieee802/help. html>.

ISO 6780, 2003. Flat pallets for intercontinental materials handling principal dimensions and tolerances. ISO Standard.

Jackson, T., Mansfield, K., Saafi, M., Colman, T., Romine, P., 2008. Measuring soil temperature and moisture using wireless MEMS sensors. Measurement, in press.

Jedermann, R., Behrens, C, Westphal, D., Lang, W., 2006. Applying autonomous sensor systems in logistics - Combining sensor networks, RFIDs and software agents. Sensors and Actuators A: Physical 132 (1), 370-375.

Maxwell, D., Williamson, R., 2002. Wireless temperature monitoring in remote systems, sensors. Sensorsmag, October 2002. <http://www.sensor smag. com/articles/1002/26/main. shtml >.

Norris, A., Saafi, M., Romine, P., 2008. Temperature and moisture monitoring in concrete structures using embedded nanotechnology/ microelectromechanical systems (MEMS) sensors. Construction and Building Materials 22 (2) (February), 111-120.

Qingshan, S., Ying, L., Gareth, D., Brown, D., 2004. Wireless intelligent sensor networks for refrigerated vehicle. In: IEEE Sixth Symposium on Emerging Technologies: Mobile and Wireless Communication, Shangai, China.

Rodrfguez-Bermejo, J., Barreiro, P., Robla, J.I., Ruiz-Garcia, L., 2007. Thermal study of a transport container. Journal of Food Engineering 80 (2), 517-527.

Ruiz-Garcia, L., Barreiro, P., Rodriguez-Bermejo, J., Robla, J.I., 2007. Monitoring intermodal refrigerated fruit transport using sensor networks: a review. Spanish Journal of Agricultural Research 5 (2).

Shih, E., Cho, S., Ickes, N, Min, R., Sinha, A., Wang, A., Chandrakasan, A., 2001. Physical layer driven protocol and algorithm design for energy-efficient wireless sensor networks. In: ACM SIGMOBILE Conference on Mobile Computing and Networking, July 2001, Rome, Italy.

Wang, N, Zhang, N, Wang, M., 2006. Wireless sensors in agriculture and food industry - recent development and future perspective. Computer and Electronics in Agriculture 50, 1-14.

Wise, K.D., 2007. Integrated sensors, MEMS, and microsystems: reflections on a fantastic voyage. Sensors and Actuators A: Physical 136 (1) (May), 39-50.

ZigBee Alliance, 2005. ZigBee Specifications, version 1.0, April 2005. $<$ http ://www. ZigBee. org >. 